\title{
The New BL Lac Candidates from the FBS
}

\author{
O. Kh. Torosyan
}

BAO,Armenia 378433; E-mail: ofelia@bao.sci.am

\begin{abstract}
Six new possible BL Lac candidates from the FBS are presented.
\end{abstract}

\section{Introduction}

The optical spectrum of BL Lac objects is the most distinguishable characteristic for them. In spite of that, there are only 10 objects classified as BL Lacs from optical surveys. Most of these objects have been selected from radio or X-ray surveys and are called, respectively, RBLs and XBLs. The number of BL Lac's has now increased by tens or hundreds on account of X-ray sources (Padovani and Giommi, 1995; Kock et al., 1996; Wolter et al., 1996; etc.).

Some Markarian galaxies have characteristics of BL Lac's: the variability and nonthermal radiation. Three of them (Mkn 180, Mkn 421, Mkn 501) are the only known Lacertids from the Markarian galaxies today. They are the most famous RBLs and XBLs simultaneously. Mkn 421 and Mkn 501 are the first extragalactic objects detected at $\mathrm{TeV}$ energies by ground-based observations (there are only 4 other such objects now, and all of them are BL Lacs); and there isn't any foundation to think that they are the exceptional ones among the $1500 \mathrm{Mkn}$ galaxies.

The completeness of these objects was estimated by the well known relation (Fig.1): $\lg N\left(m_{v}\right)=0.6 m_{v}+$ const.

The slope of this relation is about 0.57 for the relatively bright objects $\left(m_{v}<16^{m}\right)$ and only 0.3 for the objects with $16^{m}<m_{v}<17^{m}$ in the regions of the FBS survey.

\section{Selection Criteria}

The absence of emission lines in the spectra of the Markarian galaxies with starlike nuclei and E or S0 morphological typeswith may be the common selection criteria for possible candidate BL Lacs: almost all of the 34 RBLs from the $1 \mathrm{Jy}$ sample (Stickel et al., 1993); more then $94 \%$ of BL Lac host galaxies observed by the $3.6 \mathrm{~m}$ CFHT (Wurtz et al., 1996); more then $92 \%$ of BL Lac host galaxies observed by HST (Urry et al., 2000) are galaxies with E or S0 morphology. Just by these principal criteria the possible candidate BL Lacs from the catalogue of Markarian galaxies have been selected (CCAO, 1989):

- They are classified in the catalogue (CCAO, 1989) as the galaxies without emission lines (information on the emission is absent or they are specified as 
absorption galaxies as well);

- if there is information, the morphologies of the host galaxies are necessarily elliptical (E) or lenticular(S0), or any information is absent;

- the nuclei of the galaxies are classified (in Byurakan classification) as starlike (s) or semi-starlike objects (sd, ds);

- the lower bound of absolute magnitude is taken as $M_{v}=-18^{m}$ (the lower bound for the list of BL Lac objects);

- the values of visual angular sizes of the galaxies are conventionally taken as $D_{o p} \geq 10^{\prime \prime}$

\section{Results}

There are 224 objects in the catalogue of 1500 Markarian galaxies (CCAO, 1989) without emission lines. 184 of them have star-like or semi-star-like nuclei. and are possible candidate BL Lacs. Information about IR emission is absent for almost all of them (180 of 184). In contrast, all of the Sy galaxies have IR emission above the IRAS threshold. Only Mkn 501 has IR emission above the IRAS threshold of the three well-known FBS Lacertids. For nine of them, the radio fluxes are more than $100 \mathrm{mJy}$ for at least one of the four wavelengths in the 1995 catalogue of the radio survey of Markarian galaxies (Bicay, Kojoian et al., 1995) and may be classified as RBLs (Table 1). For 6 of these objects, the average radio spectral indexes are less then 0.6 , which is one of the necessary characteristics of BL Lacs (Stocke, et al., 1991).

In Table 1 are presented, respectively, the redshifts, the visual magnitudes, the absolute magnitudes, the spectral types of the nuclei by Markarian classification, the galaxies morphological types and the presence of emission (e) or absorption (a) (CCAO, 1989), the radio fluxes at $1.415 \mathrm{GHz}$ and $4.755 \mathrm{GHz}$ in $\mathrm{mJy}$ (Bicay, Kojoian, et al., 1995) as well as the radio spectral indices (Sanamian, Kandalian, 1980).

Table 1. The Possible New RBL Candidates from Mkn Galaxies

\begin{tabular}{rccclccccr}
\hline $\begin{array}{r}\text { Mkn } \\
\text { No. }\end{array}$ & $\bar{Z}$ & $m_{v}$ & $M_{v}$ & $\begin{array}{l}\text { Sp } \\
\text { (core) }\end{array}$ & $\begin{array}{c}\text { Mph } \\
\text { Host }\end{array}$ & $\begin{array}{c}\text { Sp } \\
\text { Type }\end{array}$ & $\begin{array}{c}F_{1.415} \\
(\mathrm{mJy})\end{array}$ & $\begin{array}{c}F_{4.755} \\
(\mathrm{mJy})\end{array}$ & $\alpha_{R}$ \\
\hline 11 & 0.0133 & 14.4 & -19.7 & $\mathrm{~s} 3$ & - & $\mathrm{e}$ & $\mathrm{n} / \mathrm{a}$ & 288 & 0.19 \\
${ }^{*} 180$ & 0.0458 & 15.5 & -21.1 & $\mathrm{~s} 1 \mathrm{e}$ & - & $\mathrm{BL}$ & $\mathrm{n} / \mathrm{a}$ & 227 & 0.18 \\
${ }^{4} 421$ & 0.0308 & 13.1 & -22.6 & $\mathrm{~s} 1 \mathrm{e}$ & $\mathrm{E} 1$ & $\mathrm{BL}$ & $\ldots$ & 584 & 0.15 \\
422 & 0.0316 & 14.8 & -21.0 & $\mathrm{sd} 3 \mathrm{e}:$ & - & $\ldots$ & 758 & - & - \\
452 & 0.0174 & 14.0 & -20.4 & $\mathrm{sd} 3$ & $\mathrm{SO}$ & $\mathrm{a}$ & $\ldots$ & $\leq 268$ & - \\
$* 501$ & 0.0337 & 13.7 & -22.3 & $\mathrm{~s} 2 \mathrm{e}:$ & $\mathrm{SO}$ & $\mathrm{BL}$ & $\mathrm{n} / \mathrm{a}$ & 1199 & 0.16 \\
514 & 0.0470 & 14.8 & -22.1 & $\mathrm{~s} 2$ & $\mathrm{E}$ & $\mathrm{a}$ & 228 & 157 & 0.60 \\
1007 & 0.0180 & 14.2 & -20.4 & $\mathrm{~s} 3 \mathrm{e}:$ & $\mathrm{SO}$ & $\mathrm{a}$ & $\ldots$ & 172 & 0.44 \\
1077 & 0.0229 & 16.0 & -19.8 & $\mathrm{ds} 2$ & - & $\mathrm{n} / \mathrm{a}$ & $\leq 378$ & - & - \\
\hline
\end{tabular}

Acknowledgments. I am grateful to E. Ye. Khachikian, V. O. Chavushian and R. A. Kandalian for consultation and useful advice. 


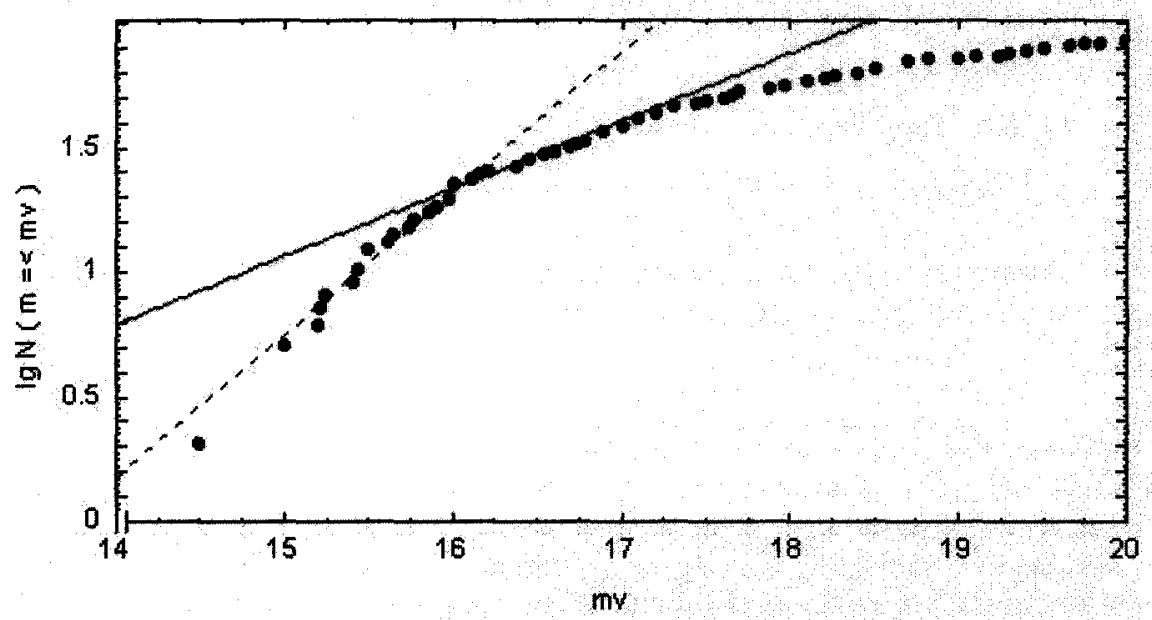

Figure 1. The relation $\lg N\left(m_{v}\right)-m_{v}$ for BL Lacs in the regions of the FBS. $y_{1}$ - dotted line; $y_{2}$ - solid line

\section{References}

Bicay, M. et al.,1995 AJ, 98, N2

FBS, 1989, CCAO, 62,3

Kock, A. et al., 1996, A\&A, 307, 745

Lipovetsky, V., 1987, CCAO, 53, 47

Mazarella, J., 1986, ApJS, 62, 751

Padovani, P., Giommi, 1995, MNRAS, 277, 1477

Petrosian, A. R., Turato, M., 1986, A\&A, 163, 26

Sanamian, V. A., Kandalian, R. A., 1980, Afz, 16, 3, 425

Stickel, M., Frid, J. W. and Kuhr, H., 1993, A\&AS, 98, 39

Stocke, et al., 1998, ApJS, 76, 813

Torosyan, O. Kh.; -in prep. (Afz)

Urry, C. M., et al., 2000, AJ, 532, 816

Wurtz R., et al., 1996, ApJS, 103, 109 